\title{
Super Resolution Reconstruction Based on Adaptive Detail Enhancement for ZY-3 Satellite Images
}

\author{
Hong Zhu ${ }^{a}$, Weidong Song ${ }^{\mathrm{a}}$, Hai Tan ${ }^{\mathrm{b}}$, Jingxue Wang ${ }^{\mathrm{a}}$, Di Jia ${ }^{\mathrm{c}}$ \\ ${ }^{\text {a }}$ School of Geomatics, Liaoning Technical University, Fuxin, Liaoning-zhuhong19890408@163.com, \\ ${ }^{\mathrm{b}}$ Satellite Surveying and Mapping Application Center of China, NO.1 Baisheng Village, Beijing- tanh@ @asmac.cn \\ ${ }^{c}$ School of Electronic and Information Engineering, Liaoning Technical University, Huludao, Liaoning-apple_jiadi@ 163.com
}

Commission VII, WG VII/6

KEY WORDS: super resolution; adaptive detail enhancement; multi-scale decomposition; image fusion

\begin{abstract}
:
Super-resolution reconstruction of sequence remote sensing image is a technology which handles multiple low-resolution satellite remote sensing images with complementary information and obtains one or more high resolution images. The cores of the technology are high precision matching between images and high detail information extraction and fusion. In this paper puts forward a new image super resolution model frame which can adaptive multi-scale enhance the details of reconstructed image. First, the sequence images were decomposed into a detail layer containing the detail information and a smooth layer containing the large scale edge information by bilateral filter. Then, a texture detail enhancement function was constructed to promote the magnitude of the medium and small details. Next, the non-redundant information of the super reconstruction was obtained by differential processing of the detail layer, and the initial super resolution construction result was achieved by interpolating fusion of non-redundant information and the smooth layer. At last, the final reconstruction image was acquired by executing a local optimization model on the initial constructed image. Experiments on ZY-3 satellite images of same phase and different phase show that the proposed method can both improve the information entropy and the image details evaluation standard comparing with the interpolation method, traditional TV algorithm and MAP algorithm, which indicate that our method can obviously highlight image details and contains more ground texture information. A large number of experiment results reveal that the proposed method is robust and universal for different kinds of ZY-3 satellite images.
\end{abstract}

\section{INTRODUCTION}

Super resolution, the process of obtaining one or more high resolution images from one or more low resolution observations, has been a very attractive research topic over the last two decades (Nasrollahi K et al, 2014; Dogiwal S R et al, 2014; Chen X J et al, 2013). In recent years, it has become one of the most active research directions in the image processing and computer vision. In remote sensing imaging applications, highquality imageries are required and high resolution images are usually desired for remote sensing images analysis and processing procedure (Yang D et al, 2015; Kaibing Z et al, 2015). Therefore, it is very useful to reconstruction high resolution images with complementary information can be increased and the quality of images can be enhanced.

With the development of space information technology, such as the domestic remote sensing satellite, navigation and positioning, satellite remote sensing image have been widely used in various fields and play an important role. In order to detect and identify some small objects accurately, the high resolution satellite images are required (Gou S et al, 2014; Zhang $\mathrm{H}$ et al, 2014; Park S J et al, 2013). However remote sensing images resolution depend on the precision of the sensor, imaging system performance improvement with expensive production costs. So to improve the image resolution from hardware aspects, the manufacturing process, systemic cost and emission load limits are bottleneck problems. Therefore we try to utilize the software, through the image processing technology to improve remote sensing image resolution, and to meet the demands, this is the remote sensing images super resolution reconstruction. Thus it can be seen that super resolution reconstruction in improve spatial resolution image technology is an effective way. According to the way of acquisition the image data, the super resolution reconstruction can be divided into: single image super resolution reconstruction and sequence of image super resolution reconstruction. And then from the information theory, whether single image or sequence images, the purpose is not only to enlarge image, but to make the results cover terrain texture details as much as possible. In that way it is significant to use the non-redundancy information from the sequence images. At present sequence of image super resolution reconstruction is based on the frequency and spatial. Methods based on frequency domain are usually using the Fouier transform and wavelet transform (S. A. Devi et al, 2012; Rasti P et al, 2014) Methods based on spatial mainly includes: interpolation method (Batz M et al, 2015; Makwana R R, et al, 2013), regularization method (Yinhui L I et al, 2015; JM Fadili et al, 2009) and the MAP method (Villena S et al, 2013; Liu C et al, 2011) and so on. The super resolution reconstruction methods have achieve some progress and breakthrough, but at the same time the algorithm with highly complexity, the model of noise simple, poor real-time performance and robustness, and the detail information of result is not remarkable. In view of the existing super resolution reconstruction methods, the super reconstruction model framework of adaptive multi-scale details enhancement is proposed in this paper, whose purpose is to achieve the results both contain micro and macro information. The paper is organized as follows. In the next section, the basic concepts from bilateral filter and multi-scale decomposition are introduced. In the third section, the proposed approach to super resolution reconstruction method is described. The approach is

Corresponding author: Weidong Song,

E-Mail: Intu_swd@163.com

This contribution has been peer-reviewed. The double-blind peer-review was conducted on the basis of the full paper. 
applied to ZY-3 satellite images in the fourth section. Conclusion about the approach is given in the fifth section.

\section{PROPOSED ALGORITHM}

In photogrammetry and remote sensing, images are often decomposed into multi scale layers. It is typically computed by applying an edge-preserving smoothing operator to the image. Most recent applications in computer graphics and computational photography use the bilateral filter (Tomasi $\mathrm{C}$ et al, 2014), popularized by Tomasi and Manduchi [1998]. It is a non-liner filter, where each pixel in the filtered result is a weighted mean of its neighbour, with the weights decreasing both with spatial distance and with difference in value. Formally, we have:

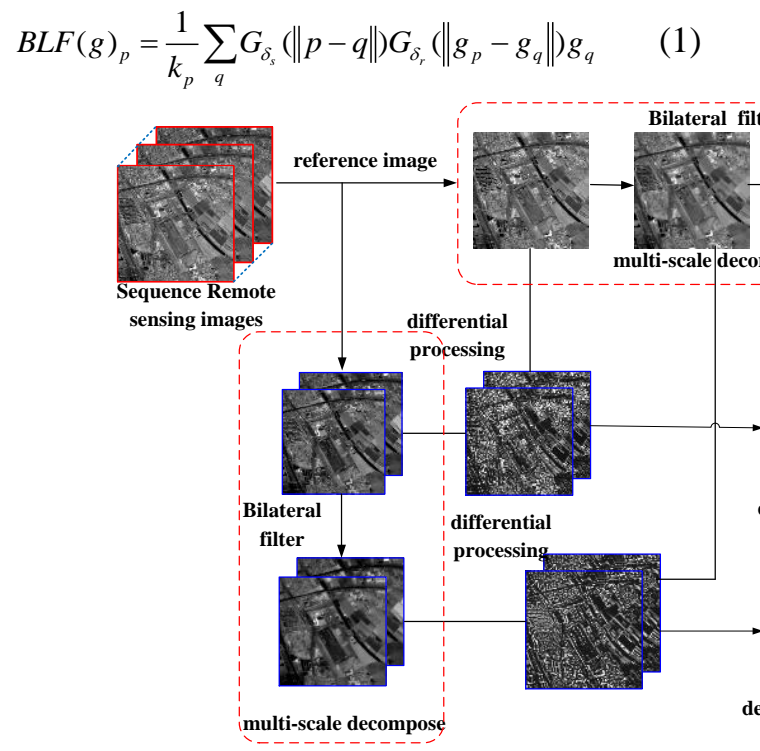

$$
k_{p}=\sum_{q} G_{\delta_{s}}(\|p-q\|) G_{\delta_{r}}\left(\left\|g_{p}-g_{q}\right\|\right) g_{q}
$$

Where $g$ is an image, and the subscripts $p$ and $q$ indicate spatial locations of pixels. The kernel functions $G_{\delta_{s}}$ and $G_{\delta_{r}}$ are typically Gaussians, where $\delta_{s}$ determines the spatial support, while $\delta_{r}$ controls the sensitivity to edges.

While the bilateral filter is quite effective at smoothing small changes in intensity while preserving strong edges, its ability to achieve progressive coarsening is rather limited. Sequence images multi-scale decomposition are used the bilateral filter, each image is decomposed into a piecewise smooth base layer and one or more detail layers. The model of multi-scale decomposition is illustrated in Figure 1. The purpose is to add the more high frequency information to the reconstruction results through adaptive detail enhancement based on multiscale decomposition.

Fig.1 Flow chart of the proposed super resolution framework

\subsection{Multi-scale Decomposition through bilateral filtering} and calculating

Given that the super resolution reconstruction image can contain more texture detail information, the bilateral filter is used to multi-scale decomposition for sequence remote sensing images in this paper. To make the notation succinct, we take Image 1 as an example, in order to construct a $(k+1)$-level decomposition. More specifically, let $u_{j}^{l}, \ldots, u_{j}^{k}$ denote progressively coarser versions of $\operatorname{Image}_{1}, u_{j}^{k}$ will serve as the base layer $I^{\text {base }}$, with the $k$ detail layers defined as

$$
d_{j}^{i}=u_{j}^{i-1}-u_{1}^{i} \text {, where } i=1, \cdots, k, u_{j}^{0}=\operatorname{Im} a g e_{j}^{i}, j=1,2, \cdots, n
$$

\subsection{Texture information Adaptive Enhancement and Fusion}

In view of the existing super resolution reconstruction method does not fully consider the problem of the detail of image texture, avoid the phenomenon of the edge blur or texture disappear in the reconstruction results. We structure the detail enhancement function which is defined as eq.(4). The function $S\left(\alpha_{i}, d_{j}^{i}\right)$ is structured which is used to strengthen of the multiscale decomposition of small and medium scale texture information.

$$
S\left(\alpha_{i}, d_{j}^{i}\right)=\left(2 /\left(1+\exp \left(-\alpha_{i} * d_{j}^{i}\right)\right)\right)-1
$$

Where $S\left(\alpha_{i}, d_{j}^{i}\right)$ is the texture detail enhancement function, it enables to enlarge small and medium-sized details of image, $\alpha$ controls the amplitude of detail, $d_{j}^{i}$ is the different scale decomposition of the sequence images. On the basis of enhancement, the different scale decomposition information is interpolated.

In order to make the algorithm more optimized based on the multi-scale decomposition, the adaptive detail enhancement function is put forward in this paper. It can avoid setting the different parameters to improve the quality of reconstruction image. The adaptive function is defined as eq.(5).

$$
X_{0}=I^{\text {base }}+\sum_{j=1}^{n} \sum_{i=1}^{k}\left(\beta_{i} \cdot S\left(\alpha_{i}, d_{j}^{i}\right)\right), 0 \leq \beta_{i} \leq 1
$$

Where $X_{0}$ is the initial super resolution reconstruction image, $I^{\text {base }}$ is the meaning of the redundant information of sequence images, $\beta_{i}$ is used to measure the weight of each detail layer, at the same time, which meets a condition: $\sum_{i=1}^{k} \beta_{i}=1$.

\subsection{Local Optimization Model}

In order to avoid the image grey value changing, better protect the image edge structures, the local optimization is used in this paper. It is defined as eq.(6).

$$
\hat{X}=\arg \min _{X}\|B X-Y\|_{2}^{2}+\mu\left\|X-X_{0}\right\|_{2}^{2}
$$

Where $Y$ is the reference image in sequence images, $B$ is the down sampling matrix, $\mu$ is the smooth scale parameter. The initial super resolution reconstruction result $\mathrm{X}_{0}$ is divided into sub-window, and down sample the sub-window. Then the sub region and the corresponding reference image are made 
difference processing, the absolute value of difference between each region is calculated. If the value is little than the preset threshold, the iterative optimization local optimization model is used. Using gradient descent optimize to solve the eq.(6), the purpose is to make the definition of the final results as far as close to the reference image and keep the sharpen edge information.

\subsection{Super Resolution Reconstruction Results Quality Evaluation Standard}

In order to evaluate the quality of reconstruction image better, we take the subjective and objective evaluation method to evaluate the quality of reconstruction image. In this paper information entropy and enhancement measure evaluation are used to evaluate the results. Information entropy is an important measure of image information index, and measure of the information distribution of the image. The greater the entropy is, the greater the result is. In other words the more information is in the results. The entropy mathematical expression is defined as eq.(7). $p_{k}$ is the frequency of the gray value of $k$ in the image, $p_{k}$ is instead of probability approximately.

$$
\text { Entropy }=-\sum_{k=1}^{M} P_{k} \log _{2} P_{k}
$$

Enhancement measure evaluation, the image is divided into $k_{l}$ $\times k_{2}$ area in this principle, the logarithmic of maximum and minimum value in sub area are calculated. $E M E$ is the local gray level image, the greater the $E M E$ value is, the much stronger local gray changed. That is to say image contains much more details, $E M E$ expression is defined as eq.(8).

$$
E M E_{k_{1}, k_{2}}=\frac{1}{k_{1}, k_{2}} \sum_{i=1}^{k_{2}} \sum_{k=2}^{k_{1}} 20 \log \frac{I_{\mathrm{max} ; k, j}^{w}}{I_{\mathrm{min} ; k, j}^{w}}
$$

Where $I_{\mathrm{max} ; k, j}^{w}$ and $I_{\mathrm{min} ; k, j}^{w}$ is the maximum and the minimum

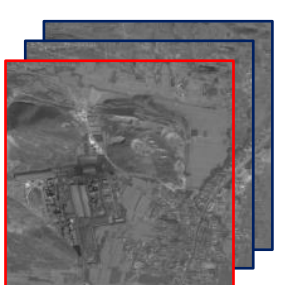

(a) Reference image

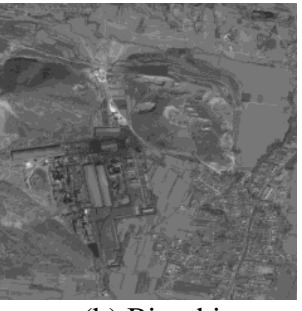

(b) Bi-cubic

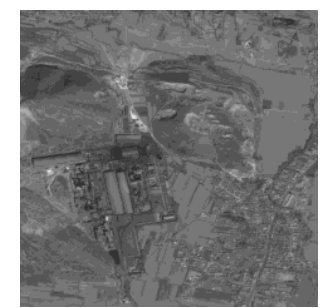

(c) TV Algorithm

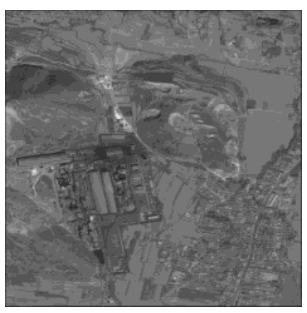

(d) MAP Algorithm

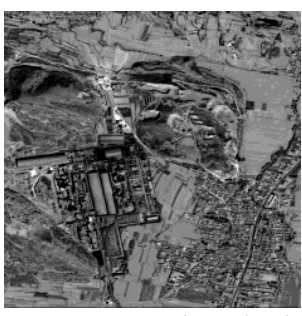

(e) Proposed method

Fig.2. Different time remote sensing image super resolution results

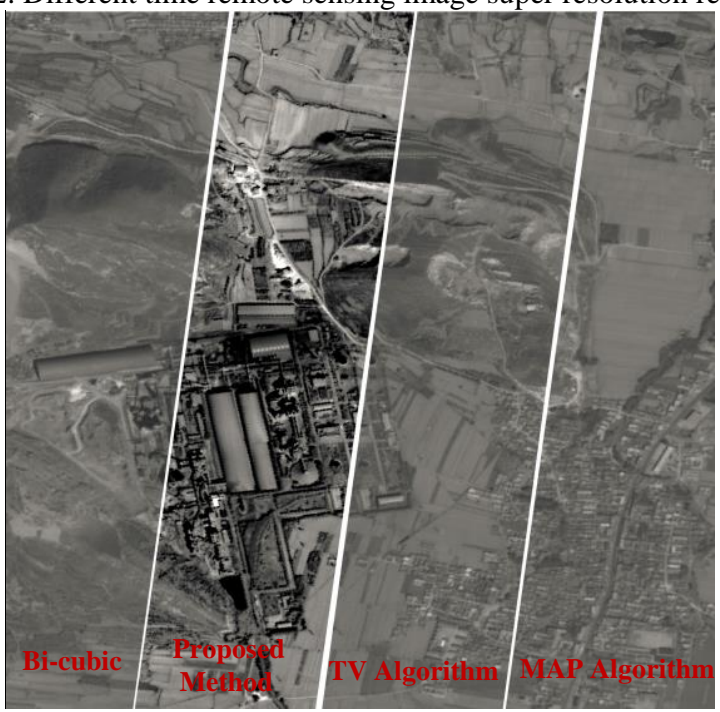


Fig.3 Synthetic image of different reconstruction methods

In experiment two, select the Three-Line-Scanner images concluding looking forwards, downwards and backwards in the same time by TDI CCD camera of ZY-3 satellite, which looking downwards resolution is $2.1 \mathrm{~m}$, the others is $3.5 \mathrm{~m}$. The images were obtained on July 10, 2013. Experimental results as shown in Figure 4, figure 4(a) is low resolution reference image with a red border, figure 4(b)-(e) are different results of super-resolution reconstruction, figure 4(e) is the reconstruction results of method in this paper, figure 5 is shown by the corresponding local amplification area of figure 4, and according to the resolution 1:1 for the reconstruction results. It is concluded that the method in this paper is much better than others, image texture information is obviously enhanced and with better visual effect.

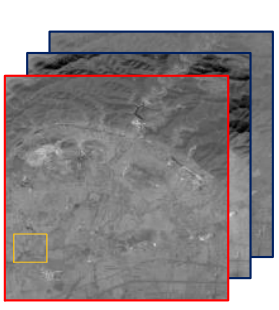

(a) Reference image

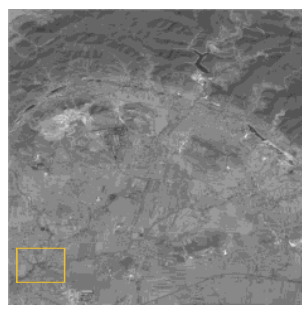

(b) Bi-cubic

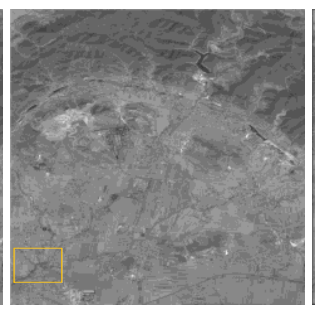

(c) TV Algorithm

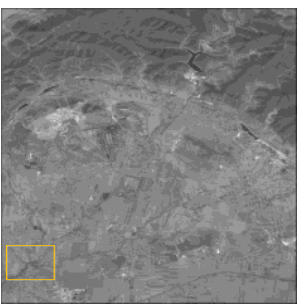

(d) MAP Algorithm

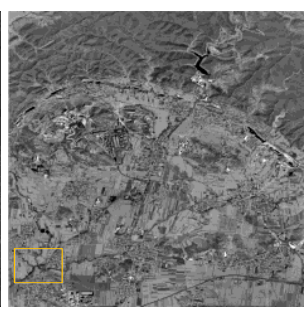

(e) Proposed method

Fig.4 Same time remote sensing image (different methods) super resolution results

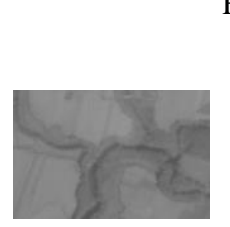

(a) Reference image

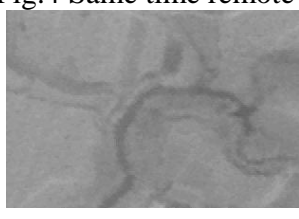

(b) Bi-cubic

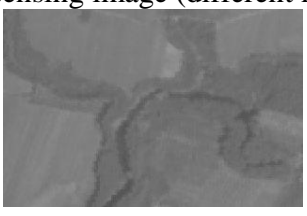

(c) TV Algorithm

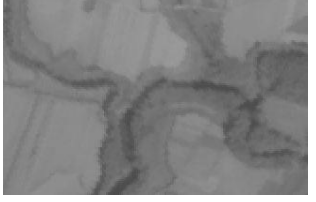

(d) MAP Algorithm

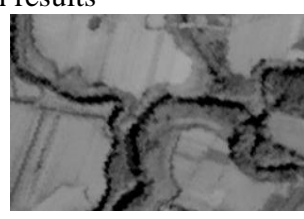

(e) Proposed method

Table 1 shows the objective evaluation of different reconstruction methods in Fig. 4(b) - (e), it is visibly to find out that the EME value is better than the reference image. Experiments show that the local details have obvious advantages, can effectively promote the overall contrast of reconstruction image, highlight detail reconstruction image. The visual effect of Fig.5(e) clearer than the Fig.5(b)-(d), the method is proposed in this paper which can improve spatial resolution at the same time keep the edge structure an enhance the details information of image.

Tab.1 Experimental results index comparison

\begin{tabular}{c|c|c|c|c|c|c}
\hline Images & Parameters & Reference images & Bi-cubic & TV Algorithm & MAP Algorithm & Propose Method \\
\hline \multirow{2}{*}{ Experiment1 } & Entropy(bits) & 6.1445 & 6.1661 & 6.1841 & 6.1936 & $\mathbf{7 . 2 4 4 6}$ \\
\cline { 2 - 7 } & EME & 3.7231 & 4.2681 & 4.7990 & 4.6909 & $\mathbf{1 4 . 2 3 0 7}$ \\
\hline \multirow{2}{*}{ Experiment2 } & Entropy(bits) & 6.2524 & 6.2526 & 6.2702 & 6.2621 & $\mathbf{6 . 9 1 4 4}$ \\
\cline { 2 - 7 } & EME & 2.8955 & 3.3455 & 3.7678 & 4.5511 & $\mathbf{1 0 . 8 6 0 0}$ \\
\hline
\end{tabular}

Because the traditional interpolation method is simple, quick and easy to implement, reconstruction results are blurred, this kind of method is difficult to effectively improve on the visual effects. Through adaptive multi-scale enhancement of super resolution reconstruction framework, the visual effect is improved after interpolation reconstruction and the expression of texture details is enriched in this paper. Compared to TV algorithm, the MAP algorithm, the proposed method can not only to keep the edge of the image, and enrich the texture information, but also to improve the visual effect significantly. Through comparing with the quantitative indicators in Tab.1, it is easy to find out the value of Entropy and EME are increased. That is, it can effectively enhance the visibility of the image detail texture, and has better robustness and universality for satellite remote sensing images.

\section{CONCLUSIONS}

The different but similar complementary information between series remote sensing images is an important source of reconstructing high-resolution remote sensing image details. Based on the characteristics of satellite remote sensing image and compensatory information between sequence images, a suitable model for remote sensing image super resolution reconstruction is put forward, and a framework of super resolution reconstruction based on adaptive multi-scale detail enhancement is proposed. That is, differential the decomposition results between the sequence and reference images, enhance small and medium size details of image adaptively, express effectively reconstruction image texture to improve the quality of reconstruction image taking into account both subjective and micro information details. In order to avoid excessive detail enhancement leading to distortion of images as a whole, this paper improves the coordination between local and overall of the results based on the local optimization model, and make them satisfy the requirement of human vision. The result from experimental data analysis shows that it is easy to find out that the entropy and EME values are promoted compared with double three interpolation, TV algorithm, and MAP algorithm. In other words, the method proposed in this paper has edge-preserving, and effectively improve the texture details. It turns out that the consistency of subjective and objective evaluation from the visual effect and quality evaluation index, enhance remote sensing image surface texture details expression effect, protect the interest area of 
reconstruction images. There is important application value and broad application prospect in the field of remote sensing.

\section{REFERENCES}

Batz M., Eichenseer A., Seiler J., et al., 2015. Hybrid superresolution combining example-based single-image and interpolation-based multi-image reconstruction approaches, IEEE International Conference on Image Processing, Quebec City, pp. 58-62.

Chen X. J., Han G. Q., Li Z., Liao X. X., 2013. Image superresolution via multi-resolution image sequence, IEEE International Conference on Wavelet Analysis and Pattern Recognition, pp. 178-183.

Devi S. A., Vasuki A., 2012. Image super resolution using Fourier-wavelet transform, International Conference on Machine Vision and Image Processing, Taipei, pp.109-112.

Dogiwal S R., Shishodia Y., Abhay U., 2014. Super Resolution Image Reconstruction using Wavelet Lifting Schemes and Gabor filters, Confluence The Next Generation Information Technology Summit, Nodia, pp. 625-630.

Fadili J. M., Gabriel P., 2009. Total variation projection with first order schemes, IEEE International Conference on Image Processing. Cairo, pp. 1317-1320.

Gou S., Yang S., 2014. Remote Sensing Image SuperResolution Reconstruction Based on Nonlocal Pairwise Dictionaries and Double Regularization. IEEE Journal of Selected Topics in Applied Earth Observations \& Remote Sensing, 7(12), pp.4784-4792.

Liu C., Sun D., 2011. A Bayesian approach to adaptive video super resolution, IEEE Conference on Computer Vision \& Pattern Recognition, Providence, RI, pp. 209-216.

Li Y. H., L X. Q., Yu H. F., 2015. Sequence images superresolution reconstruction based on $\mathrm{L}_{-} 1$ and $\mathrm{L} \_2$ mixed norm, Journal of Computer Applications, 35(3), pp.840-843.

Makwana R. R., Mehta N. D., 2013. Single Image SuperResolution VIA Iterative Back Projection Based Canny Edge Detection and a Gabor Filter Prior, International Journal of Soft Computing \& Engineering, 3(1), pp.379-384.

Nasrollahi K., Moeslund T. B., 2014. Super-resolution: a comprehensive survey, Machine Vision \& Applications, 25(6), pp.1423-1468.

Park S. J., Lee O. Y., Kim J. O., 2013. Self-similarity based image super-resolution on frequency domain, Signal and Information Processing Association Annual Summit and Conference, Kaohsiung, pp. 1-4.

Rasti P., Lusi I., Demirel H., et al., 2014. Wavelet Transform Based New Interpolation Technique for Satellite Image Resolution Enhancement, IEEE International Conference on Aerospace Electronics and Remote Sensing, Technology, pp. 185-188.

Salvador V., Miguel V., 2013. Bayesian combination of sparse and non-sparse priors in image super resolution, Digital Signal Processing, 23(2), pp.530-541.

Tomasi C., Manduchi R., 2014. Bilateral Filtering for Gray and Color Images, International Conference on Computer Vision. IEEE Computer Society, pp.839 - 846.
Yang D. Q., Li Z. M., Xia Y. T. Chen Z. Z., 2015. Remote sensing image super-resolution: Challenges and approaches, IEEE International Conference on Digital Signal Processing, Singapore, pp. 196-200.

Zhang H., Yang Z., Zhang L., 2014. Super-Resolution Reconstruction for Multi-Angle Remote Sensing Images Considering Resolution Differences. Remote Sensing, 6(1), pp. 637-657.

Zhang K. B., Tao D. C., 2015. Learning multiple linear mappings for efficient single image super-resolution. IEEE Transactions on Image Processing, 24(3), pp.846-861. 